KN01.24

Acta Cryst. (2005). A61, C1

\section{A Theoretician's View of Crystallography}

Roald Hoffmann, Department of Chemistry and Chemical Biology, Cornell University, Ithaca NY 14853, USA. E-mail: rh34@cornell.edu

Whether thinking qualitatively or quantitatively, molecular structure, most often determined by crystallography, is central to the aims of quantum chemists. This long-time consumer and observer of crystal structures will talk about the conceptual terrain between theorists and crystallographers. Among the topics broached will be the complex problem of what is a chemical bond, experimental and "chemical" standard deviations, and the working out of secondary, donor-acceptor interactions in the solid state.

Keywords: molecular structure, secondary interactions, chemical bond

\section{KN02.24}

Acta Cryst. (2005). A61, C1

Structure and Recognition in the BARS/CtBP-dependent Transcription Regulation

Martino Bolognesi, Marco Nardini, Department of Biomolecular Sciences and Biotechnology and INFM, University of Milano, Milano, Italy. E-mail: martino.bolognesi@unimi.it

BARS/CtBP3 is a dual function protein acting as acyl-transferase in the Golgi apparatus (supporting membrane reshaping and vesicle traffic) [1], and as transcription co-repressor, in the nucleus, through the interaction with several enzymatic partners (e.g. histone deacetylases, HDACs). BARS/CtBP3 is based on a 3-domain structure, hosting a classical dehydrogenase fold [2]. Regulation of the two activities is achieved through competitive binding of $\mathrm{NAD}(\mathrm{H}) /$ acyl-CoA, association equilibria, SUMO-ylation, and eventually through recognition of specific sequence motifs in the interacting partners. Binding of specific transcription factors to each subunit in the dimeric BARS/CtBP3, through a PXDLS sequence motif, is considered one of the basic mechanisms to recruit HDACs, and modify the chromatin structure, with ensuing transcription repression [2]. Structural considerations and mutant analyses indicate that different recognition sites are present on BARS/CtBP3 surface, in keeping with its pivotal role within a nuclear protein complex hosting more than twenty different proteins.

[1] Weigert R., Silletta M.G., Spano S., Turacchio G., Cericola C., Colanzi A., Senatore S., Mancini R., Polishchuk E.V., Salmona M., Facchiano F., Burger K.N.J., Mironov A., Luini A., Corda D., Nature, 1999, 402, 429. [2] Nardini M., Spano S., Pericola C. Pesce A., Massaro A., Millo E., Luini A., Corda D., Bolognesi M., EMBO J., 2003, 22, 3122.

Keywords: biomolecular recognition, enzyme function, transcription regulation

\section{KN03.24}

Acta Cryst. (2005). A61, C1

Ion Pumping by $\mathrm{Ca}^{2+}$-ATPase of Sarcoplasmic Reticulum

Chikashi Toyoshima, Institute of Molecular and Cellular Biosciences, The University of Tokyo,Tokyo, Japan. E-mail: ct@iam.u-tokyo.ac.jp

$\mathrm{Ca}^{2+}$-ATPase of skeletal muscle sarcoplasmic reticulum (SERCA1a) is an integral membrane protein of $110 \mathrm{~K}$ and the best characterised member of the P-type (or E1/E2-type) ion translocating ATPases. It transports $2 \mathrm{Ca}^{2+}$ and counter-transports $2 \sim 3 \mathrm{H}^{+}$per ATP hydrolysed. SERCA1a consists of 10 transmembrane helices, 3 cytoplasmic domains (A, actuator; $\mathrm{N}$, nucleotide binding; $\mathrm{P}$, phosphorylation) and small lumenal loops [1]. In E1, transmembrane $\mathrm{Ca}^{2+}$-binding sites have high affinity and face the cytoplasm; in E2, the binding sites have low affinity and face the lumen of sarcoplasmic reticulum (extracellular side). Actual transfer of bound $\mathrm{Ca}^{2+}$ is thought to take place between E1P and E2P. We have determined the crystal structures of this enzyme in 5 different states [2], namely, $\mathrm{Ca}^{2+}$-bound $\mathrm{E} 1 \cdot 2 \mathrm{Ca}^{2+}, \mathrm{Ca}^{2+}$-unbound but thapsigargin bound E2(TG), $\mathrm{Ca}^{2+}-$ and AMPPCP-bound E1-AMPPCP, $\mathrm{Ca}^{2+}-, \mathrm{ADP}-$ and $\mathrm{AlF}_{\mathrm{x}}$ bound $\mathrm{E} 1 \cdot \mathrm{AlF}_{\mathrm{x}} \cdot \mathrm{ADP}$, and $\mathrm{Ca}^{2+}$-unbound but $\mathrm{MgF}_{4}{ }^{2-}$ bound $\mathrm{E} 2 \cdot \mathrm{MgF}_{4}{ }^{2-}$, where $\mathrm{MgF}_{4}{ }^{2-}$ and $\mathrm{AlF}_{\mathrm{x}}$ work as stable analogues of phosphate. Detailed comparisons of these structures show that very large rearrangements of cytoplasmic domains and transmembrane helices take place, and that ATP, phosphate, $\mathrm{Ca}^{2+}$ and $\mathrm{Mg}^{2+}$ are the principal modifiers of the domain interactions.

[1] Toyoshima C., Inesi G., Ann. Rev. Biochem., 2004, 73, 269. [2] Toyoshima C., Nomura H., Tsuda T., Nature, 2004, 432, 361.

Keywords: ion pump, membrane proteins, inhibitors

\section{KN04.24}

Acta Cryst. (2005). A61, C1

Neutrons and $\mathrm{X}$-rays for Microstructure and Strain/Stress Analysis in Materials

Walter Reimers, Technical University Berlin, Metallic Materials, Ernst-Reuter-Platz 1, D-10587 Berlin, Germany. E-mail: walter.reimers@tu-berlin.de

The microstructure and the strain/stress distribution in engineering materials can be adjusted as a function of the materials processing technology and its parameters. Since the microstructure and the strain/stress distribution in components are responsible for macroscopical properties, e.g. yield strength fatigue life, the property profile of components can be optimised in view of the in-service condition. The optimation requires the detailed knowledge about microstructural parameters like phase composition, grain sizes, dislocation densities, texture, strains/stresses and their relationship to the macroscopical properties. The various contributions to this field using X-ray and neutron diffraction are presented. Special emphasis is put to the rapid development of the microstructural and strain/stress analysis due to the increasing use of synchrotron X-radiation which enables new measuring techniques but also in-situ analysis of microstructural alterations and strain/stress developments. Examples are given for selected material systems like Mg-alloys and brass alloys.

Keywords: strain/stress, X-ray diffraction, Mg-alloy

\section{KN05.25}

Acta Cryst. (2005). A61, C1

Single-particle Diffraction

David Sayre, Dept. of Physics and Astronomy, Stony Brook University, Stony Brook NY 11794, USA. E-mail: sayre@xray1.physics.sunysb.edu

$\mathrm{X}$-ray crystallography is approaching the time when it will be able to image single small objects without the need to aggregate multiple copies into a crystal. The emerging technique is quite close to that of $\mathrm{x}$-ray crystallography, but replaces the crystal's large amplification of the diffracted signal by the use of high-intensity $\mathrm{x}$-ray sources and techniques for minimizing the effects of radiation damage. The subject will be illustrated by describing progress in our project aiming at $3 \mathrm{D}$ $10 \mathrm{~nm}$-resolution imaging of a single quick-frozen yeast cell.

The talk will serve as an introduction to Microsymposium MS22, which will describe additional ongoing work, including projects aimed at atomic-resolution imaging of single macromolecules and macromolecular assemblies by the use of femtosecond-length pulsed $\mathrm{x}$-ray sources.

Keywords: crystallography without crystals, X-ray diffraction microscopy, yeast cell

\section{KN06.25}

Acta Cryst. (2005). A61, C1-C2

Elucidating the Structures of Nanoporous Microcrystals

Lynne B. McCusker, Laboratory of Crystallography, ETHHönggerberg, CH-8093 Zürich, Switzerland. E-mail: lynne.mccusker@mat.ethz.ch

With their stable and open aluminosilicate framework structures, zeolites and their analogs have proven to be ideally suited for intriguingly diverse applications. Their pore openings, which range from 0.25 to $2.0 \mathrm{~nm}$, have molecular diemensions; their void space can reach up to $50 \%$ of the total volume; they are crystalline with well-defined and ordered pores; and their framework structures are anionic or neutral. These features are exploited in their use as 


\section{KEYNOTE LECTURES}

molecular sieves, as shape-selective catalysts, as dessicants, as ionexchangers, and as hosts in advanced materials. A zeolite's suitability for a specific application is highly dependent upon its structure, so structure analysis is an intrinsic part of zeolite science. However, because most zeolites can only be prepared in polycrystalline form, standard methods of structure analysis cannot be applied.

Over the years, zeolite crystallographers have devised a number of different methods to overcome or circumvent this problem. Initially, physical model building based on information from various sources was the only option available. Interestingly enough, this is probably still the most powerful tool in the zeolite crystallographers toolbox, but it requires experience, talent and intuition. As computing capacity has increased, however, algorithms for automating the model building process have been created. At the same time, methods for improving the quality of reflection intensities extracted from powder diffraction patterns have been devised, and this in turn has allowed single-crystal methodology to be applied with greater success. An overview of some of the more recent developments in this field will be presented.

Keywords: zeolites, powder diffraction, structural analysis software

\section{KN07.25}

Acta Cryst. (2005). A61, C2

Structure Analysis of Modulated Crystals: Trends and Tendencies Václav Petř́ček, Michal Dušek, Institute of Physics, Praha, Czech Republic. E-mail: petricek@fzu.cz

The superspace theory as developed by DeWolff, Jansen \& Janner [1] gave to crystallographers a unique tool for generalization of structural analytical methods to be especially applicable to modulated structures. In many cases the structure analysis can now be performed almost routinely [2]. The superspace approach can also be used to find a systematic way of describing whole families of related structures [3]. The use of CCD and imaging plate systems changed considerably sensitivity of data collection for modulated structures and therefore a need for further improvement of the methods is obvious. The modulation of more complicated systems cannot be efficiently described as series of harmonic functions. Special discontinuous functions already introduced for $3+1$ dimensions [4] are to be generalized to $3+2$ and $3+3$ superspace.

Recently modulations have been found in complicated organic structures including proteins. This opens a various new problems concerning efficiency of methods used for solution and refinement of modulated structures. New techniques such as maximum entropy [5] and charge flipping methods [6] give us a good chance to make such a generalization.

[1] Wolff de P.M., Janssen T, Janner A., Acta Cryst., 1981, A37, 625. [2] Petrríček V., Dušek M. Z.,Kristallogr., 219, 692. [3] Perez-Mato J.M., ZakhourNakhl M., Weill F., Darriet J. J., Mat. Sci., 1999, 9, 2795. [4] Petříček V., van der Lee A., Evain M., Acta Cryst., 1995, A51, 529. [5] Smaaalen van S., Palatinus L., Schneider M., Acta Cryst., 2003, A59, 459. [6] Palatinus L. Acta Cryst., 2004, A60, 604.

Keywords: modulated crystal structures, structure analysis, superspace theory

\section{KN08.25}

Acta Cryst. (2005). A61, C2

High Throughput Technologies in Structural Biology

Raymond C. Stevens, Department of Molecular Biology, The Scripps Research Institute, 10550 North Torrey Pines Road, La Jolla, CA 92037 USA. E-mail: stevens@scripps.edu

During the past few years, progress has been made in developing high throughput technologies for protein cloning, expression, purification, crystallization, crystal imaging, and synchrotron beamline data collection. Recently, we have been able to miniaturize, automate and parrallelize the structural biology processes significantly using nanoliter volume technologies (see http://stevens.scripps.edu/ webpage/htsb for examples). Accordingly, significantly smaller amounts of materials can be used at all steps, and more parallel experiments can be engineered (genetic and mechanical) within the same space and time constraints. The next phase of this effort includes integration and improved system processing. A description of these technology developments, current status, and examples will be described.

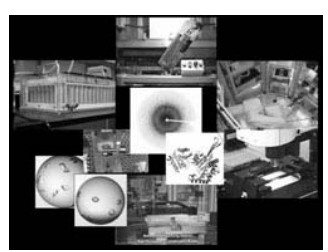

Figure 1. Sample of technologies that have been created in the past few years that include robotics systems for expression, protein purification, imaging, and analysis.

Acknowledgements: I am grateful for the cooperativity of the combined effort of researchers from the Joint Center for Structural Genomics (NIH PSI GM062411), JCIMPT (NIH Roadmap Initiative P50GM073197), Genomics Institute of the Novartis Research Foundation, The Scripps Research Institute, and Syrrx.

Keywords: structural genomics, structure based drug discovery, high throughput structural biology

KN09.25

Acta Cryst. (2005). A61, C2

Structure Solution of Pharmaceutical Compounds from Powder Diffraction Data

Peter W. Stephens, Department of Physics and Astronomy, Stony Brook University, Stony Brook, NY 11794 USA. E-mail: pstephens@stonybrook.edu

A significant part of contemporary medicine is based on the discovery and development of drugs, which are often molecules of ten to thirty non-hydrogen atoms. It is important to know the crystal structures of drug compounds and candidates for various reasons: fundamental understanding of structure and bonding vis-à-vis physiological action, the physical and chemical properties of polymorphs which are frequently encountered in drugs, and the relevance of polymorphism to patent protection and limits thereon. As it happens, many of these materials are available only as powders, and therefore any structural information must be obtained from powder diffraction.

Advances in instrumentation and data analysis techniques, both commercial and in the public domain, are proving equal to the task. However, judging from the literature, structure determination from powder data SDPD is still an obscure art, practiced by relatively few crystallographers. This is despite the outreach activities of a significant number of the innovators of SDPD, who have been working to develop and promulgate powder techniques.

I will review the state of the art and present some new results, such as the structures of chloramphenicol palmitate polymorphs.

Keywords: ab-initio powder structure determination, pharmaceutical structure determination, polymorphism

\section{KN10.25}

Acta Cryst. (2005). A61, C2-C3

Quasicrystal Structure Analysis. The State of the Art

Akiji Yamamoto, Advanced Materials Laboratory, NIMS, Namiki, Tsukuba, Japan. E-mail: Yamamoto.Akiji@nims.go.jp

The quasicrystal is an aperiodic solid showing Bragg peaks with noncrystallographic symmetry. It is recently clarified that the structure of quasicrystals can be analyzed by using a newly developed direct method and a structure refinement which is based on a higherdimensional cluster model. They are equally applicable to decagonal and icosahedral quasicrystals since all quasicrystals seem to consist of some atom clusters (or building units)[1].

For the initial model building, the low-density elimination method (LDEM) is efficient [2]. This gives rough shape and size of occupation domains (OD) of a quasicrystal, which specify the location of atoms in a higher-dimensional space.

An initial model for the structure refinement is obtained from the rough ODs determined by LDEM by considering atom clusters, which are included in its crystal approximants. The distribution of atom clusters can not, however, be determined uniquely because of the existence of random phason strain, which is seen in all quasicrystals. This is usually inferred from high-resolution electron microscopy images or simply assumed based on a quasiperiodic tiling. The 